References:

[1] Richette P et al. 2016. Ann Rheum Dis 2017;76:29-42.

[2] Nielsen SM et al. Ann Rheum Dis 2017 76(11):1870-1882.

[3] Gudbergsen $\mathrm{H}$ et al. BMJ 2019. 71-2.

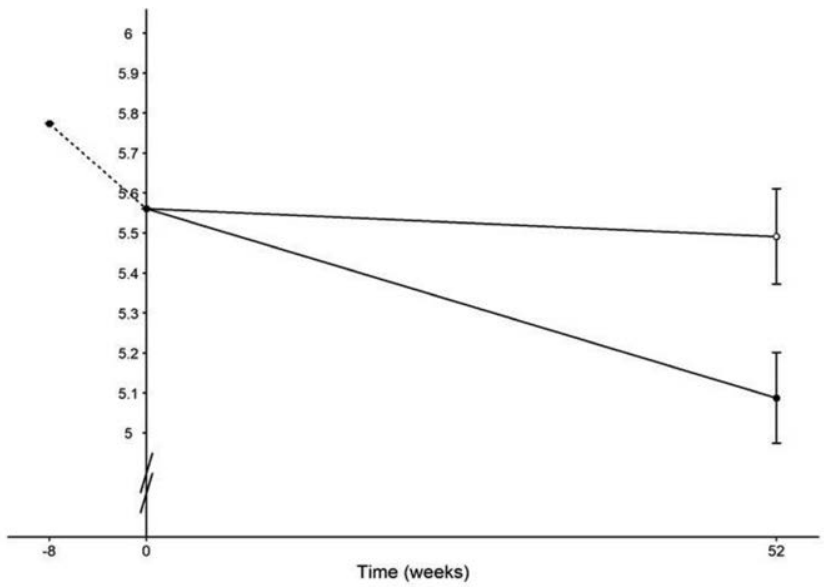

Figure 1: Serum urate at different timepoints: Estimates are unadjusted means at week -8 and 0 (dotted line; $\mathrm{n}=155$ ) and least square means estimates from an ANCOVA model (adjusted for stratification factors, i.e. sex, age category, obesity class as well as the level of the outcome at baseline) for data at 52 weeks (solid lines; $n=134)$. Solid points at week 52 indicate the liraglutide group $(n=69)$ and open points indicate the placebo group $(n=65)$. The error bars indicate SE.

Disclosure of Interests: Kristian Zobbe: None declared, Sabrina Mai Nielsen: None declared, Robin Christensen: None declared, Anders Overgaard: None declared, henrik gudbergsen Speakers bureau: Pfizer 2016, Marius Henriksen: None declared, Henning Bliddal Grant/research support from: received research grant fra NOVO Nordic, Consultant of: consultant fee fra NOVO Nordic, Lene Dreyer: None declared, Lisa Stamp: None declared, Filip Krag Knop Shareholder of: Minority shareholder in Antag Therapeutics Aps, Grant/research support from: AstraZeneca, Gubra, Novo Nordisk, Sanofi and Zealand Pharma, Consultant of: Amgen, AstraZeneca, Boehringer Ingelheim, Carmot Therapeutics, Eli Lilly, MSD/Merck, Mundipharma, Novo Nordisk, Sanofi and Zealand Pharma., Speakers bureau: AstraZeneca, Bayer, Boehringer Ingelheim, Eli Lilly, Medlmmune, MSD/Merck, Mundipharma, Norgine, Novo Nordisk, Sanofi and Zealand Pharma., Lars Erik Kristensen Consultant of: UCB Pharma (Advisory Board), Sannofi (Advisory Board), Abbvie (Advisory Board), Biogen (Advisory Board), Speakers bureau: AbbVie, Amgen, Biogen, Bristol-Myers Squibb, Celgene, Eli Lilly, Gilead, Forward Pharma, Janssen Pharmaceuticals, MSD, Novartis, Pfizer, and UCB Pharma

DOI: 10.1136/annrheumdis-2020-eular.3088

\section{OP0173 \\ IMMUNOMODULATION CO-THERAPY WITH PEGLOTICASE: DATABASE TRENDS 2014-2019}

B. Lamoreaux ${ }^{1}$, M. Francis-Sedlak ${ }^{1}$, K. Svensson ${ }^{1}$, R. Holt ${ }^{1} .{ }^{1}$ Horizon Therapeutics, Lake Forest, United States of America

Background: Pegloticase is a PEGylated biologic therapy for patients with uncontrolled gout who have not improved on or could not tolerate conventional urate-lowering therapies. ${ }^{1}$ All biologics have the ability to engender anti-drug antibodies (ADAs) and it is known that some patients given pegloticase develop ADAs that cause them to stop treatment prior to recieving a complete course of therapy. ${ }^{2-3}$ In other rheumatic autoimmune diseases, DMARDs such as methotrexate or azathioprine are used as standard of care to prevent the development of ADAs to biologics. These DMARDs often allow patients to remain on biologic therapies longer and recieve the full therapeutic benefits while minimizing adverse events. ${ }^{4}$ While pegloticase has been used traditionally as monotherapy, recent case series have demonstrated the therapeutic benefit of immunomodulator co-administration, allowing more patients to receive a full course of pegloticase therapy. ${ }^{5-6}$ Little has been published on how widespread this practice is and whether it has changed over time.

Objectives: To examine medical claims database from 2014-2019 for trends in immunomodulating therapies being co-prescribed with pegloticase.

Methods: An IQVIA claims database (November 2014 to October 2019) representing 1.3 billion claims, covering 30 million patients diagnosed with gout or CKD, was utilized to search for patients who had received pegloticase. Patients who had recieved pegloticase were classified as having been on an immunomodulating co-therapy if they were prescribed methotrexate or azathioprine within 60 days before or after initiation of their first pegloticase infusion.
Results: We found relatively steady low rates of immunomodulation co-therapy with pegloticase from 2014 through 2018 ranging from 1\% in 2016 to $4 \%$ in 2018 (Figure 1). In 2019 however, the proportion of pegloticase patients that were co-treated with methotrexate or azathioprine therapy increased to $15 \%$. Most patients were started on immunomodulating therapy 20 days before to 10 days after initiation of pegloticase. Methotrexate was the more frequently used immunomodulaton co-therapy as compared to azathioprine.

Figure 1: Proportion of pegloticase patients receiving immunomodulation therapy by year

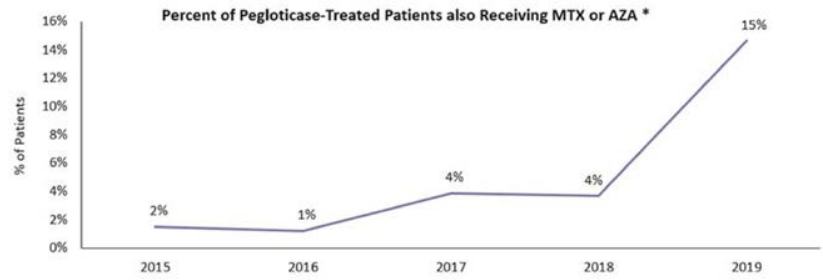

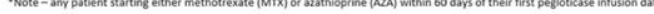

Conclusion: We found evidence of a relatively dramatic increasing initiation of immunomodulation therapy with pegloticase beginning soon after a November 2018 presentation of a case series which demonstrated improved response rates of pegloticase when co-administered with methotrexate. These data indicate that clinicians began to more frequently employ a strategy of DMARD co-treatment with pegloticase in 2019 to improve response rates to this important gout medicine.

References:

[1] Sundy JS, et al. JAMA 2011;306:711-20.

[2] Abeles AM. Arthritis Research \& Therapy 2014, 16:112

[3] Strand V, et al. BioDrugs 2017; 31:299-316.

[4] Krieckaert CL, et al. Arthritis Res Ther 2010;12:217.

[5] Botson J and Peterson J. Ann Rheum Dis. 2019; 78: A1289

[6] Bessen SY, et al. Semin Arthritis Rheum. 2019;49:56-61.

Disclosure of Interests: Brian LaMoreaux Shareholder of: Horizon Therapeutics, Employee of: Horizon Therapeutics, Megan Francis-Sedlak Shareholder of: Horizon Therapeutics, Employee of: Horizon Therapeutics, Karl Svensson Shareholder of: Horizon Therapeutics, Employee of: Horizon Therapeutics, Robert Holt Shareholder of: Horizon Therapeutics, Employee of: Horizon Therapeutics

DOI: 10.1136/annrheumdis-2020-eular.3893

\section{\begin{tabular}{|l|l|l|l}
\hline OP0174 POLYDATIN PREVENTS CALCIUM PYROPHOSPHATE \\
\hline
\end{tabular} CRYSTAL-INDUCED ARTHRITIS IN MICE}

F. Oliviero ${ }^{1}$, F. Galuppini ${ }^{1}$, A. Scanu ${ }^{1}$, P. Galozzi ${ }^{1}$, V. Lazzarin ${ }^{1}$, P. Sfriso ${ }^{1}$, G. Ravagnan ${ }^{2}$, R. Ramonda ${ }^{1}$, P. Spinella ${ }^{1}$, L. Punzi ${ }^{3}$, G. Pennelli ${ }^{1}$, R. Luisetto ${ }^{1} .{ }^{1}$ University of Padova, Padova, Italy; ${ }^{2}$ Institute of Translational Pharmacology CNR, Rome, Italy; ${ }^{3}$ Ospedale SS Giovanni e Paolo, Venezia, Italy

Background: Acute calcium pyrophosphate (CPP) crystal-induced inflammation is characterized by the massive release of cytokines and pro-inflammatory mediators and, from a clinical point of view, pain and limited joint function. Contrary to the precipitation of urate crystals that can be prevented through the use of hypouricemic drugs, there is no pharmacological therapy that can prevent the formation of pyrophosphate crystals.

Polydatin (PD), a natural precursor of resveratrol, is a stilbenoid mainly contained in grape juice and bark of Polygonum Cuspidate. Its antioxidant, anti-inflammatory and immunomodulating properties have been demonstrated in several experimental models. We have recently shown that this compound is able to prevent the inflammatory response to pathogenic crystals in vitro (1)

Objectives: The aim of this study was to assess the anti-inflammatory preventing effect of polydatin in the mouse model of acute crystal-induced arthritis. Methods: A suspension of sterile CPP crystals $(0.3 \mathrm{mg} / 20 \mu \mathrm{L}$ PBS) have been injected intra-articularly (i.a.) into one ankle joint of Balb/c mice under isoflurane anesthesia. Animals were randomized in 5 groups: 1- CPP injection, 2- CPP + PD, 3- CPP + colchicine (control drug), 4- CPP + vehicle (control. N 1), 5- PBS injection (control N. 2). Polydatin and colchicine were administered by gavage (respectively $40 \mathrm{mg} / \mathrm{kg}$ and $1 \mathrm{mg} / \mathrm{kg}$ in $200 \mu \mathrm{L} \mathrm{PBS} / \mathrm{EtOH} /$ glucose) at 24, 15 and $1 \mathrm{~h}$ before and 1, 6 and $24 \mathrm{~h}$ after (prophylactic model) or 1, 6 and $24 \mathrm{~h}$ after (therapeutic model) i.a. injection of CPP crystals.

Ankle swelling was measured at different time points using a precision caliper. After $48 \mathrm{~h}$ (peak of the acute phase) mice were euthanized and blood and ankle 